(Aus der allgemeinen Poliklinik, Basel.)

\title{
Respirationsversuche am Menschen im nüchternen Zustand und nach Zufuhr verschiedener Eiweisskörper.
}

Von

WilheIm coefiter.

I.

Nach Zufuhr von Eiweisskörpern oder Kohlehydraten findet beim Menschen im normalen Ernährungszustand stets eine Steigerung der Sauerstoftabsorption und der Kohlensäureabgabe statt. Diese Steigerung beginnt ziemlich unmittelbar nach der Nahrungsaufnahme, um nach mehr oder weniger langer Zeit wieder auf den Nüchternwert herunter zu gehen.

Die Steigerung des Respirationsstoffwechsels wird von den verschiedenen Autoren verschieden gedeutet. Die Zun tz-Mering'sche Schule ${ }^{1}$ ) führt die sofort nach der Nahrungsaufnahme sich zeigende Steigerung auf die "Verdauungsarbeit" zurück. Rubner ${ }^{1}$ ) veranschlagt den Beitrag der Verdauungsarbeit an dieser Steigerung als sehr gering. Nach ihm kommt jedem Nahrungsstoff eine „spezifischdynamische Wirkung" zu. Von fast alien Autoren wird ausserdem angenommen, dass ein Teil der aufgenommenen Nahrung direkt verbrannt und damit sofort vom Organismus verwertet wird.

Bis vor wenigen Jahren waren jeweils nur die verschiedenen Hauptgruppen der Nahrungsstoffe (Eiweisskörper, Kohlehydrate, Fette) miteinander verglichen worden. Es wurde angenommen, dass die verschiedenen Eiweisskörper resp. die verschiedenen Kohlehydrate qualitativ und quantitativ die gleichen Veränderungen im respiratorischen Stoffwechsel bedingten, doch lagen keine experimentellen

1) Zuntz und Mering, Inwietern beeinflusst Nahrungszufuhr die tierischen Oxydationsprozesse? Pflüger's Arch. Bd. 32 S. 173. 1888.

2) Rubner, Die Gesetze des Energieverbrauchs bei der Ernährung. 190\% Pflüger's Arehiv für Plysiologie. Ba. 147. 
Prüfungen dieser Frage vor. [V oit $\left.{ }^{1}\right)$ ]. Versuche, die einen Teil dieses Problems betreffen, liegen in der Publikation von Falta, Grote und Staehelin ${ }^{2}$ ) vor. Diese Autoren fütterten einen Hund mit je gleichen Mengen (120 g) Casein, Glutencasein und hydrolysiertem Kasein zusammen mit einer Standardkost, bestehend aus Pferdefleisch, Schweinefett und Fleischextrakt. Sie kamen zu dem Resultat, dass ein wesentlicher Unterschied zwischen dem Einfluss dieser Eiweisskörper auf die Kalorienproduktion, sowie auf den Sauerstoffverbrauch und die Kohlensäureabgabe nicht besteht. Nach anderen Gesichtspunkten hin hatte unter anderen St a ehelin ${ }^{3}$ ) die Wirkung der vegetarischen Diät auf den Menschen studiert. Bedeutende Unterschiede in der Wirkung animalischer und vegetabilischer Kostform fand er nicht: Körpertemperatur, Muskelleistung, Pulsfrequenz, Blutdruck, Viskosität des Blutes usw. zeigten keine bedeutenden Verschiedenheiten.

Nur die Diurese wurde durch Fleisch und Eier ausgesprochen erhöht. Sowoinl Wasser als auch Kochsalz und vielleicht auch die stickstoff haltigen Endprodukte des Stoffwechsels erfuhren eine vermehrte Ausscheidung, die vom Autor auf eine Wirkung der Extraktivstoffe zurückgeführt wurde. Dies bestärkt unsere Annahme, dass zur Untersuchung der Wirkung von Eiweisskörpern oder Kohlehydraten auf den Stoffwechsel diese Stoffe möglichst frei von allen Zutaten aufgenommen werden müssen.

Stoffwechselversuche zur Vergleichung der Wirkung verschiedener, reiner Eiweisskörper beim Menschen sind unseres Wissens noch nicht ausgeführt worden. Der scheinhar naheliegende Einwand, man überschreite mit der Zufuhr reiner Eiweisskörper ohne jede Zutat und Zubereitung die Grenzen des Physiologisehen, ist unbegründet: die von uns gewählten Körper (Casein, Edestin) sind leicht einzunehmen und werden ohne jede Beschwerde ertragen. Ausserdem beweisen, wie später zu ersehen sein wird, die Versuche mit solchen Eiweisskörpern, dass letztere vom Organismus ausgezeichnet resorbiert und verwertet werden. Dann liegt es in der Natur jedes Versuches,

1) Vo it, Die Ernährung. Herrmann 's Handb. d. Phys. Bd. 6 S. 104. 1881.

2) Falta, Grote und Stähelir, Versuche über den Kraft- und Stoffwechsel und den zeitlichen Ablauf der Zersetzungen unter dem Einfluss verschiedener Ernährung beim Hund. Hofmeister's Beitr. Bd. 9 S. 333.

3) Staehelin, Untersuchungen über die vegetarische Diät usw. Zeitschr. f. Biol. Bd. 49 S. 199.1907. 
möglichst eindeutige klare Bedingungen zu schaffen, um aus den Resultaten Schlüsse ziehen zu können.

Bis vor wenigen Jahren fehlten auch vergleichende Untersuchungen über die Wirkung verschiedener reiner Kohlehydrate auf den Gaswechsel. Die ersten Untersuchungen, die diese Frage betreffen, stammen von Johannsson ${ }^{1}$ ) aus dem Jahre 1908. Bei einer Prüfung der Hypothese von der gleichen Wirkung der verschiedenen Kohlehydrate kam dieser Forscher zu dem auffallenden Ergebnis, dass z. B. Lävulose eine doppelt so grosse Steigerung der Kohlensäureproduktion hervorruft wie dieselbe Dose Dextrose. Im Mittel von 11 bzw. 10 Bestimmungen bei verschiedenen Individuen betrug die Steigerung der Kohlensäureabgabe nach einer Dose von

$$
\begin{aligned}
& 50 \mathrm{~g} \text { Dextrose . . . . . . + 7,8 g CO} \\
& 50 \mathrm{~g} \text { Lävulose . . . . . + }+13,3 \mathrm{~g} \mathrm{CO} \text {. }
\end{aligned}
$$

Der Widerspruch dieses Befundes mit der früheren Annahme dürfte, wie schon oben erwähnt, zum Teil darin liegen, dass Johannsson reine Körper verwendete, während in den meisten Arbeiten von $\operatorname{Rubner^{2}}$ ), Magnus-Levy ${ }^{3}$ ) u. a. die Zufuhr aus sehr verschiedenen zusammengesetzten Speisen bestand (Dextrose, Reis, Reis + Zucker, Reis + Zucker + Fleisch + Fleischextrakt usw.). Weiter dürfte ein grosser Unterschied darin zu suchen sein, dass Johannsson die Veränderungen des Gaswechsels unmittelbar nach der Nahrungsaufnahme, bis maximal 12 Stunden danach berücksichtigt, während die anderen Autoren 24 stündige und noch längere Perioden in Betracht zogen.

Die Untersuchungen der letzten Jahre haben ergeben, dass die verschiedenen Eiweisskörper in ihrer chemischen Konstitution und in ihren Beziehungen zum Organismus (z. B. Abbauprodukte) sich sehr verschieden verhalten können. Es dürfte daher von Interesse sein, auch hier zu prüfen, wie sie sich zum respiratorischen Gaswechsel verhalten.

Der Zweck der vorliegenden Versuche ist, den Einfluss von verschiedenen, möglichst reinen Eiweisskörpern auf

1) Johannsson, Untersuchungen über den Kohlehydratstoffwechsel. Skand. Arch. f. Physiol. Bd. 21. 1908.

2) Rubner, l. c.

3) Magnus-Levy, Über die Grösse des respiratorischen Gaswechsels. Pflüger's Arch. Bd. 55 S. 1. 1894. 
den Gaswechsel des Menschen zu untersuchen. Sie beziehen sich ausschliesslich auf die Veränderungen des Stoffwechsels, welche sich unmittelbar nach der Nabrungsaufnahme abspielen. Sie wurden nur beim Menschen im nüchternen Zustand, $d$. h. bei normalem Glykogenvorrat ausgeführt. Als Grundlage für die Versuche dienten Nüchternversuche an denselben Versuchspersonen.

Da der Nüchternwert ${ }^{1}$ ) (Grundumsatz) als Grundlage für die Berechnung der Stoffwechselveränderung dienen soll, haben wir uns bemüht, denselben möglichst genau zu erhalten.

Zur Methodik: Alle Versuche wurden an zwei Personen ausgefuhrt, welche in Respirationsversuchen dieser Art geübt sind. Die eine Versuchsperson (B) (Gig on) war zur Zeit der letzten Versuche 28 Jahre alt, $181 \mathrm{~cm}$ gross, das Gewicht schwankte zwischen 74,2 und 75,6 kg. Die andere Versuchsperson (A) (Loeffler) war zur Zeit der Versuche 24 Jahre alt, $173 \mathrm{~cm}$ gross;' das' Gewicht betrug $70,6-71,5 \mathrm{~kg}$.

Die Versuche wurden bei vorsätzlicher Muskelruhe ${ }^{2}$ ) ausgefübrt. Die Versuchsperson war meist wach, zeitweise schlafend. Unkontrollierte Muskelbewegungen konnten mit Sicherheit ausgeschlossen werden, da die einzelnen Perioden eines Versuches nie eine halbe Stunde überdauerten und zwischen denselben Pausen von 15-30 Minuten eingeschaltet wurden. Die Pausen wurden dazu benützt, die exspirierte Luft mit atmosphärischer Luft derart zu ver dünnen, dass bei den Analysen der $\mathrm{CO}_{2}$-Gehalt $z$ wischen 0,8 und 1,2\% schwankte. Zur Kontrolle der Mischung wurden sofort nach der Verdünnung Proben je aus den oberen, mittleren und unteren Partien des Gasometers abgefüllt. Es ergaben sich für Kohlensänre:

$\begin{array}{rccc} & \text { obere Partie } & \text { mittlere Partie } & \text { untere Partie } \\ \text { I. } & 0,927 \% & 0,920 \% & 0,926 \% \\ \text { II. } & 0,815 \% & 0,815 \% & - \\ \text { III. } & 1,122 \% & - & 1,128 \%\end{array}$

Nach diesen Ergebnissen ist also die Mischung völlig homogen.

Gleich nach der Mischung erfolgte die Entnahme der Luftproben, die über Quecksilber aufbewahrt wurden. Die Versuchsperson lag während der ganzen Versuchszeit auf einem Liegestuhl, der auch während der Pausen nicht verlassen wurde. Sämtliche Untersuchungen mit Ausnahme von einigen an Gigon wurden mittels eines Respirationsapparates ausgeführt, der nach dem Muster eines von

1) Johannsson, Über die Tagesschwankungen des Stoffwechsels usw. Skand. Arch. Bd. 8 S. 85 ff. - Magnus-Levy, Physiologie des Stoffwechsels. In v. Noorden's Handb. Bd. 1 S. 222. 1906.

2) Jaquet, Der respiratorische Gaswechsel. Ergebn. d. Physiol. Bd. 2 S. 457. 1903. (Die Versuchsperson nalım Kückenlage ein unter jeder Vermeidung von Muskelspannungen.) 
Jaquet konstruierten Apparates gebaut ist. Er gleicht dem von Speck ${ }^{1}$ ) vor ca. 30 Jahren benutzten; er hat die gleichen Vor- und Nachteile wie ein $Z \mathbf{u n t z}$ Geppert'scher Apparat. Als Aufnahmebehälter für die Exspirationsluft dient ein genau geeichtes Spirometer mit zylindrischer Glocke von 150 Liter Inhalt, die über Wasser abgesperrt ist. Die grösste Fehlerquelle bei solchen Apparaten liegt bekanntlich darin, dass bei verschiedenen Höhen der Spirometerglocke die Druckverhältnisse sich ändern. Dieser Fehler wird in unserem Apparat durch ein sehr genau konstruiertes exzentrisches Rad, über das das Gegengewicht der Glocke läuft, beseitigt. Zur Regelung der Zu- und Abfubr der Luft von der Versuchsperson verwendeten wir anfangs Metallventile, dann Müller'sche Wasserventile, da diese einen sicheren Abschluss gewähren und eine genauere Kontrolle gestatten. Der Anschluss der Versuchsperson an die Leitung erfolgte durch ein Kautschukmundstück, das durch eine elastische Binde am Munde fixiert wurde. Den Nasenverschluss machten wir anfangs wie in den Zuntz'schen Versuchen mit einer Klemme. Später zogen wir es vor, die Nasenlöcher mit feuchter Watte zu verschliessen. Mit Leukoplaststreifen lässt sich darüber bequem und sicher ein luftdichter Abschluss erzielen.

Zur Berechnung sei erwähnt, dass die Luft im Spirometer als mit Wasserdampf gesättigt angesehen werden musste.

Zur Kontrolle des Apparates diente eine kleine Lampe, die mit Petroleum gefüllt war, dessen Zusummensetzung folgende Werte ergab.

$0,1122 \mathrm{~g}$ liefern $0,3527 \mathrm{~g} \mathrm{CO}_{2}+0,1898 \mathrm{~g} \mathrm{H}_{2} \mathrm{O}$. Die Kontrollversuche gaben folgende Werte.

Tabelle I.

\begin{tabular}{l|c|c|c|c|c}
\hline Datum & Dauer & $\begin{array}{c}\text { Durch- } \\
\text { geflossene } \\
\text { Luft } \\
\text { Liter }\end{array}$ & $\begin{array}{c}\mathrm{CO}_{2} \\
\text { gefunden } \\
\mathrm{g}\end{array}$ & $\begin{array}{c}\mathrm{CO}_{2} \\
\text { berechnet } \\
\mathrm{g}\end{array}$ & Fehler \\
$\mathrm{g}$
\end{tabular}

Wie aus der Tabelle ersichtlich, ergeben die Kontrollen gut übereinstimmende Werte. $\mathrm{Zu}$ ihrer Beurteilung darf natürlich nicht eine prozentuale Berechnung angewendet werden. Nach diesen Zahlen kommt die Genauigkeit unseres Apparates derjenigen anderer Apparate (Jaquet, Tigerstedt) gleich.

Die bei einzelnen auch bier verwerteten Versuchen gebrauchten Apparate waren der von Tigerstedt und Sondén ${ }^{2}$ ) und der von Jaquet ${ }^{3}$ ) in der

1) Speck, Physiologie des menschlichen Atems nach eigenen Untersuchungen. 1892.

2) Sondén und Tigerstedt, Untersuchungen über die Respiration und den Gesamtstoffwechsel des Menschen. Skandin. Arch. f. Physiol. Bd. 6 S. 1. 1895.

3) Jaquet, Ein neuer Apparat zur Untersuchung des resp. Stoffwechsels beim Menschen. Verhandl. der naturforsch. Gesellsch. Basel Bd. 15 S. 252. 1903. 
Baslex medizinischen Klinik. Die Luftanalysen wurden in dem Apparat von Pettexs on- $\mathrm{H}$ oeg land ausgeführt. $\left(\mathrm{CO}_{2}\right.$-Absorption mit Kalilange; $\mathrm{O}_{2}$-Absorption mit Pyrogallol). Die Zusammensetzung der atmosphärischen Luft wurde am Ende eines jeden Versuches bestimmt und für die Berechnung der betreffenden Versuche verwertet. Die Stickstoffbestimmung erfolgte nach Kjeldahl. Für die Berechnung der Gasvolumina wurden die Landoltschen Tabellen benützt. Die Versuche begannen frühestens 9-12 Stunden nach der letzten Mahlzeit, die stets beträchtlich eingeschränkt wurde. Der Organismus war also sicher im nüchternen Zustand. Bei den Versuchen mit Nahrungszufuhr wurde am Anfang der Beobachtung der bestimmte Nahrungsstoff in einmaliger Dosis mit beliebiger Quantität Wasser genossen. Die Aufnahme der Respirationsiuft begann jeweils gleich nach Schluss der Nahrungsaufnahme.

\section{Nïchternversuche (Grundumsatz).}

Die folgenden Versuche entsprechen dem Grundumsatz bei Individuen mit gewöhnlichem also noch ziemlich reichlichem Glykogenvorrat (nicht bei solchen im Hungerzustand). Es stehen von Gigon Nüchternwerte, die nach drei verschiedenen Methoden bestimmt sind, zur Verfügung.

Wie aus Tabelle II (S. 203) ersichtlich ist, ergeben die Nüchternversuche gut übereinstimmende Werte. Bei A erhalten wir als Mitte] vol acht Bestimmungen einen Wert für $\mathrm{CO}_{2}$ von $20,70 \mathrm{~g}$ und für $\mathrm{O}_{2}$ von $18,92 \mathrm{~g}$ pro Stunde. Der mittlere Fehler der Einzelbeobachtung beträgt für $\mathrm{CO}_{2} \pm 1,09$, für $\mathrm{O}_{2} \pm 0,61$. Diese Werte beziehen sich auf eine Periode von $1^{1 / 2}$ Jahren. Während dieser Zeit hat sich also der Grundumsatz nicht geändert. Bei B haben die Untersuchungen mit dem $J$ aquet'schen und dem T igerstedt'schen Apparat einen Gaswechsel im Nüchternzustand von ca. $23,3 \mathrm{~g} \mathrm{CO}_{2}$ und $21,0 \mathrm{~g} \mathrm{O}_{2}$. Die Versuche am Spirometerapparat lieferten ein Mittel von 21,15 $\mathrm{g} \mathrm{CO}_{2}$ und $20,0 \mathrm{~g} \mathrm{O}_{2}$. Dieses etwas niedrigere Mittel lässt sich zum grössten Teil darauf zurückfübren, dass die Hautatmung bei der letzteren Versuchsanordnung nicht berücksichtigt werden kann.

Die Untersuchungen erstrecken sich bei Gigon auf einen Zeitraum von mehr als 4 Jahren. Diese Resultate, die mit drei verschiedenen Apparaten erhalten worden sind, sowie die gute Übereinstimmung der Nüchternwerte bei mir sind ein sicherer, wenn auch indirekter Beweis für die Zuverlässigkeit unseres Apparates.

Die Versuchsperson hat vor den Nüchternversuchen ihre übliche Arbeit getan. 
Tabelle II.

\begin{tabular}{|c|c|c|c|c|c|}
\hline Datum & $\begin{array}{l}\text { Versuchs- } \\
\text { person }\end{array}$ & $\begin{array}{c}\mathrm{CO}_{\mathrm{a}} \text { Aus- } \\
\text { seheidung } \\
\mathrm{g}\end{array}$ & $\begin{array}{l}\mathrm{O}_{\mathrm{g}} \text {-Ver- } \\
\text { brauch } \\
\text { g }\end{array}$ & $\frac{\mathrm{CO}_{2}}{\mathrm{O}_{2}}$ & Apparat \\
\hline $\begin{array}{l}\text { 10. Okt. } 1910 \\
\text { 14. Nov. } 1910 \\
\text { Dezbr. } 1911 \\
\text { Januar } 1912\end{array}$ & $\begin{array}{c}\text { Loeffler }(\mathrm{A}) \\
" \\
"\end{array}$ & $\begin{array}{l}21,92 \\
21,48 \\
19,06 \\
19,62 \\
19,97 \\
21,10 \\
21,82 \\
20,33 \\
\end{array}$ & $\begin{array}{l}18,01 \\
20,99 \\
18,09 \\
18,77 \\
18,50 \\
18,97 \\
18,97 \\
19,06 \\
\end{array}$ & $\begin{array}{l}0,848 \\
0,744 \\
0,766 \\
0,753 \\
0,793 \\
0,898 \\
0,836 \\
0,776\end{array}$ & $\begin{array}{cc}\text { Poliklinik. Spirometer } \\
", & " \\
" & " \\
" & " \\
" & " \\
" & "\end{array}$ \\
\hline $\begin{array}{l}\text { Mittel . . } \\
\text { Mittlerer Fehl } \\
\text { beobachtung }\end{array}$ & der Einzel- & $\begin{array}{l}\mathbf{0 , 7 0} \\
\pm 1,09\end{array}$ & $\begin{array}{r}15,92 \\
\pm 0,61\end{array}$ & $\begin{array}{r}0,793 \\
- \\
\end{array}$ & $\begin{array}{l}- \\
-\end{array}$ \\
\hline $\begin{array}{l}\text { Septbr. } 1907 . \\
\text { Oktober } 1907 \\
\text { Novbr. } 1908 . \\
\text { April } 1910 \\
\text { Oktober } 1910 \\
\text { Septbr. } 1910 .\end{array}$ & $\begin{array}{c}\left.\operatorname{Gig} \circ \mathrm{n}^{1}\right)(\mathrm{B}) \\
" \\
" \\
" \\
" \\
"\end{array}$ & $\begin{array}{l}22,5 \\
23,8 \\
23,8 \\
22,7 \\
21,6 \\
20,8\end{array}$ & $\begin{array}{l}- \\
21,6 \\
20,4 \\
19,5 \\
20,5\end{array}$ & $\begin{array}{l}- \\
\overline{0} \\
0,799 \\
0,811 \\
0,796 \\
0,740\end{array}$ & $\begin{array}{l}\text { Tigerstedt } \\
\text { Jaquet, med. Klinik. } \\
\text { Poliklinik. Spirometer } \\
" \#\end{array}$ \\
\hline
\end{tabular}

Unsere Versuche ergeben, dass der Grundumsatz für ein und dasselbe Individuum Jahre hindurch ganz auffallend konstant bleibt. Die Beteiligung der einzelnen Körperstoffe am Grundumsatz bleibt also auch die gleiche.

Die Annahme, welche noch öfters gemacht wird, dass im nüchternen Zustand vorwiegend Fett verbraucht wird, muss sehr vorsichtig aufgenommen werden. Es werden nicht mehr als ca. $40-50 \%$ des Umsatzes von Fett bestritten, der Rest verteilt sich ungefähr zu gleichen Teilen auf Eiweiss und Kohlehydrate. Erwähnenswert ist es vielleicht auch, dass der Grundumsatz nicht mehr als 20 bis 26 Kalorien pro Kilogramm in 24 Stunden beträgt. Diese Zahl ist niedriger als das vou $\mathrm{V}_{0 i t^{2}}$ ) aufgestellte Kalorienminimum für den ruhenden erwachsenen Menschen.

Der Nüchternwert ist auch unabhängig von der Tageszeit. Einzelne Bestimmungen wurden am Tage, andere in der Nacht ausgeführt. Ebenso ist der Grundumsatz unabhängig vom Wach- oder Schlafzustand der Versuchsperson.

1) Gigon, Über einige Fragen des Stoffwechsels und der Ernährung. Münchener med. Wochenschr. 1911 S. 1343.

2) Berechnet nach Voit: Die Ernährung. In Herrmann's Handb. d. Physiol. Bd. 6 S. 531. 
Versuchsperson A hat, wie zu erwarten war, etwas niedrigere Nüchternwerte als B, indem sie ca. $4 \mathrm{~kg}$ leichter und ca. $8 \mathrm{~cm}$ kleiner ist.

$$
\text { T abelle II a }
$$

Grundumsatz pro Stunde.

\begin{tabular}{|c|c|c|c|c|c|c|c|}
\hline \multirow[b]{2}{*}{ Autor } & \multicolumn{3}{|c|}{ Verbraucht } & \multicolumn{3}{|c|}{ Der Umsatz besteht aus } & \multirow{2}{*}{$\begin{array}{l}\text { Kalorien } \\
\text { pro Kilo- } \\
\text { gramm in } \\
24 \text { Stdn. }\end{array}$} \\
\hline & $\begin{array}{c}\text { Eiweiss } \\
\mathrm{g}\end{array}$ & $\begin{array}{c}\text { Fett } \\
\mathrm{g}\end{array}$ & $\begin{array}{l}\text { Kohle- } \\
\text { hydrat } \\
\text { g }\end{array}$ & $\begin{array}{c}\text { Eiweiss } \\
\%\end{array}$ & $\begin{array}{l}\text { Fett } \\
0 \%\end{array}$ & $\begin{array}{c}\text { Kohle-- } \\
\text { hydrate } \\
0 \%\end{array}$ & \\
\hline $\begin{array}{l}\text { Magnus-Lery') } \\
\text { Staebelin } 1 \text { ). } \\
\text { Gigon }{ }^{1} \text { ). } \\
\text { Voitt). } \\
\text { Loeffler: }\end{array}$ & $\begin{array}{l}2,25 \\
2,81 \\
3,69 \\
5,7 \\
3,5\end{array}$ & $\begin{array}{l}4,6 \\
2,71 \\
3,71 \\
2,1 \\
3,58\end{array}$ & $\begin{array}{c}2,2 \\
4,68 \\
4,46 \\
14,6 \\
3,17\end{array}$ & $\begin{array}{l}15,0 \\
20,3 \\
22,0 \\
22,6 \\
24,0\end{array}$ & $\begin{array}{l}70,0 \\
44,5 \\
51,0 \\
19,2 \\
55,0\end{array}$ & $\begin{array}{l}1.5,0 \\
35,2 \\
27,0 \\
58,2 \\
21,0\end{array}$ & $\begin{array}{l}26,6 \\
20,0 \\
22,5 \\
35,7 \\
21,6\end{array}$ \\
\hline
\end{tabular}

III. Stoffwechsel bei Zufuhr von Eiweiss.

Zur Prüfung des Einflusses des Eiweisses auf den Stoffwechsel haben wir als animalisches Eiweiss Kasein gewählt, als Pflanzeneiweiss Edestin.

Das Kasein stellt ein grawweisses Pulver dar, das deutlich nach Käse schmeckt; das Edestin ist ein sehr leichtes, hellgelbes Pulver. Beide Körper lassen sich leicht einnehmen. Beschwerden sind niemals aufgetreten.

Im Mittel erhielten wir einen Stickstoffgehalt für die von uns benützten Präparate:

Kasein $12,85 \% \mathrm{~N}$; Edestin $16,50 \% \mathrm{~N}$.

Kase in ${ }^{3}$ ) ist ein Nukleoalbumin, das aus Milch gewonnen wird. Edestin wird aus Hanfsamen dargestellt und gehört zu den Globulinen. In ihrem Aufbau aus Aminosäuren differieren die beiden Körper ganz wesentlich. Die wichtigsten Unterschiede sind aus folgender Zusammenstellung ersichtlich. ${ }^{3}$ )

1) Zitiert nach Gigon, l. c.

2) Berechnet nach Voit, Die Ernährung. In Herrmann's Handb. d. Physiol. Bd. 6 S. 531.

3) Abderhalden, Lehrb. d. physiol. Chemie. 


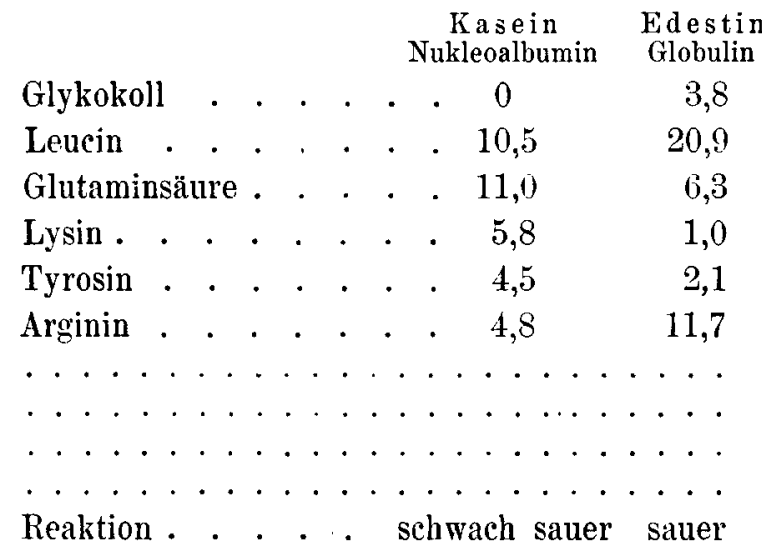

In den Versuchen wurde das Kasein resp. Edestin in einmaligen Dosen von $50 \mathrm{~g}$ nach der oben angegebenen Weise genommen.

Tabelle III ergibt die Resultate der Kaseinversuche:

Tabelle III.

Gaswechsel pro $1 / 2$ Stunde nach Zufuhr von $50 \mathrm{~g}$ Kasein.

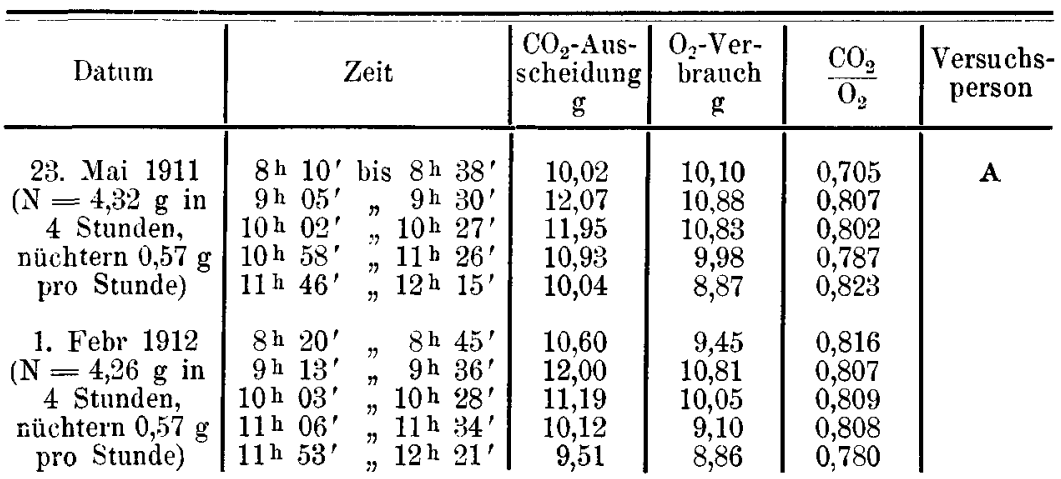

Tabelle IV.

Steigerung des Gaswechsels nach Zufuhr von $\mathbf{0 0}$ g Kasein.

\begin{tabular}{|c|c|c|c|c|}
\hline Datum & Zeit & $\begin{array}{c}\mathrm{CO}_{2} \text {-Aus- } \\
\text { scheidung } \\
\mathrm{g}\end{array}$ & $\begin{array}{c}\mathrm{O}_{2^{-}} \\
\text {Verbrauch } \\
\mathrm{g}\end{array}$ & $\begin{array}{l}\text { Versuchs- } \\
\text { person }\end{array}$ \\
\hline 23. Mai 1911 & 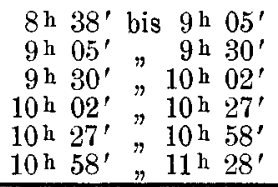 & $\begin{array}{r}9,64 \\
10,05 \\
12,80 \\
9,95 \\
11,75 \\
10,77\end{array}$ & $\begin{array}{r}9,45 \\
9,08 \\
11,58 \\
9,03 \\
10,76 \\
9,99\end{array}$ & A \\
\hline Daner . . . & $\begin{array}{l}2 \mathrm{~h} 50^{\prime} \\
\text { Nüchternwerte } \\
\text { Steigerung. . }\end{array}$ & $\begin{array}{r}64,96 \\
58,65 \\
+6,31\end{array}$ & $\begin{array}{r}59,89 \\
53,55 \\
+6,34\end{array}$ & \\
\hline
\end{tabular}




\begin{tabular}{|c|c|c|c|c|}
\hline Datum & Zeit & $\begin{array}{l}\mathrm{CO}_{2} \text {-Aus- } \\
\text { scheidung } \\
\mathrm{g}\end{array}$ & $\begin{array}{c}\mathrm{O}_{2^{-}} \\
\text {Verbrauch } \\
\mathrm{g}\end{array}$ & $\begin{array}{c}\text { Versuchs- } \\
\text { person }\end{array}$ \\
\hline 1. Februar 1912 & 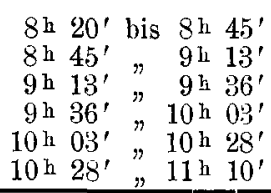 & $\begin{array}{r}8,84 \\
10,55 \\
9,20 \\
10,43 \\
9,32 \\
14,08\end{array}$ & $\begin{array}{r}7,87 \\
9,45 \\
8,29 \\
9,40 \\
8,29 \\
13,64\end{array}$ & A \\
\hline Daner . . . & $\begin{array}{l}2^{\mathrm{h}} 50^{\prime} \\
\text { Nüchternwerte } \\
\text { Steigerung. . } \\
\text { Mittel . . . }\end{array}$ & $\begin{array}{r}62,42 \\
58,65 \\
+3,77 \\
+5,01\end{array}$ & $\begin{array}{r}56,94 \\
53,55 \\
+3,39 \\
+4,57\end{array}$ & \\
\hline
\end{tabular}

Tabelle V.

Gaswechsel pro Stunde nach Zufuhr ron 50 g Kasein ${ }^{1}$.

\begin{tabular}{|c|c|c|c|c|c|}
\hline Datum & Zeit & $\begin{array}{c}\mathrm{CO}_{2} \text {-Aus- } \\
\text { scheidung } \\
\mathrm{g}\end{array}$ & $\begin{array}{l}\mathrm{O}_{2} \text {-Ver- } \\
\text { branch } \\
\mathrm{g}\end{array}$ & $\begin{array}{l}\mathrm{CO}_{2} \\
\mathrm{O}_{2}\end{array}$ & $\begin{array}{c}\text { Versuchs- } \\
\text { person }\end{array}$ \\
\hline $\begin{array}{c}\text { 4. 5. Dez. } 1908 \\
\text { (Harn-N }=6,75 \mathrm{~g} \\
\text { in } 10 \text { Stunden } \\
\text { nüchtern } 0,59 \mathrm{~g} \\
\text { pro Stunde) }\end{array}$ & 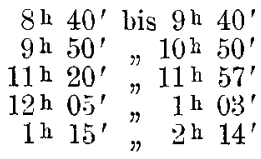 & $\begin{array}{l}24,59 \\
26,77 \\
23,80 \\
24,16 \\
22,13\end{array}$ & $\begin{array}{l}22,43 \\
23,11 \\
23,36 \\
21,74 \\
18,65\end{array}$ & $\begin{array}{l}0,797 \\
0,807 \\
0,741 \\
0,808 \\
0,891\end{array}$ & 13 \\
\hline $\begin{array}{c}\text { 17.-18. April 1910 } \\
\text { (Harn-N }=4,20 \mathrm{~g} \\
\text { in } 5 \text { Stunden } \\
\text { nüchtern } 0,59 \mathrm{~g} \\
\text { pro Stunde) }\end{array}$ & 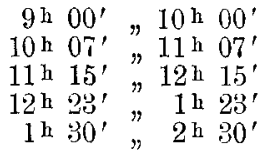 & $\begin{array}{l}22,65 \\
24,10 \\
24,88 \\
25,45 \\
22,29\end{array}$ & $\begin{array}{l}22,26 \\
21,77 \\
22,32 \\
23,67 \\
-\end{array}$ & $\begin{array}{l}0,740 \\
0,805 \\
0,810 \\
0,781 \\
-\end{array}$ & \\
\hline
\end{tabular}

Tabelle VI.

Steigerung des Gaswechsels nach Zufulhr ron 50 g Kasein.

\begin{tabular}{|c|c|c|c|c|}
\hline Datum & Zeit & $\begin{array}{c}\mathrm{CO}_{2} \text {-Aus- } \\
\text { scheidung } \\
\mathrm{g}\end{array}$ & $\begin{array}{l}\mathrm{O}_{2-} \\
\text { Verbranch } \\
\text { g }\end{array}$ & $\begin{array}{l}\text { Versuchs- } \\
\text { person }\end{array}$ \\
\hline 4.-5. Dez. 1908 & 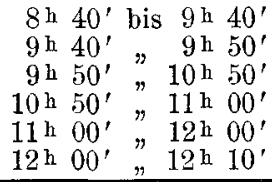 & $\begin{array}{r}24,59 \\
4,28 \\
26,77 \\
4,21 \\
23,80 \\
3,99\end{array}$ & $\begin{array}{r}22,43 \\
3,79 \\
23,11 \\
3,87 \\
29,36 \\
\mathbf{3}, 75\end{array}$ & B \\
\hline Dauer & $\begin{array}{l}3^{1 / 2} \text { Stunden } \\
\text { Nüchternwert. } \\
\text { Zunahme. . }\end{array}$ & $\begin{array}{r}87,64 \\
81,75 \\
+5,89\end{array}$ & $\begin{array}{r}80,31 \\
73,69 \\
+6,62\end{array}$ & \\
\hline
\end{tabular}

1) Gigon, Über den Einfluss der Nahrungsaufnahme auf den Gaswechsel und Energieumsatz. Pflüger's Arch. Bd. 140 S. 1. 1911. 


\begin{tabular}{|c|c|c|c|c|}
\hline Datum & Zeit & $\begin{array}{l}\mathrm{CO}_{\mathrm{g}} \text {-Aus- } \\
\text { scheidung } \\
\mathrm{g}\end{array}$ & $\begin{array}{c}\mathrm{O}_{\mathrm{g}^{-}} \\
\text {Verbrauch } \\
\mathrm{g}\end{array}$ & $\begin{array}{l}\text { Versuchs- } \\
\text { person }\end{array}$ \\
\hline 17.-18.April1910 & 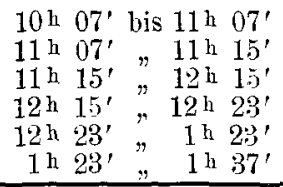 & $\begin{array}{r}24,10 \\
3,90 \\
24,83 \\
3,21 \\
25,45 \\
5,40 \\
\end{array}$ & $\begin{array}{r}21,77 \\
2,94 \\
22,32 \\
3,06 \\
2,67 \\
\quad .36 \\
\end{array}$ & B \\
\hline ... & $\begin{array}{l}\text { 31/2 Stunden } \\
\text { Nüchternwert . } \\
\text { Zunahme.. } \\
\text { Mittel. . . . }\end{array}$ & $\begin{array}{r}86,24 \\
81,75 \\
+4,49 \\
+\mathbf{5}, 19\end{array}$ & $\begin{array}{r}79,12 \\
73,69 \\
5,43 \\
+\mathbf{6 , 0 3}\end{array}$ & \\
\hline
\end{tabular}

In sämtlichen Tabellen haben wir die Zeit angegeben, welche genau der Dauer der einzelnen Versuchsperioden entsprach. Um vergleichbare Werte zu erhalten, sind dagegen die Werte in Gramm für die Kohlensäureausscheidung und den Sauerstoffverbrauch auf 30 Minuten resp. 1 Stunde gerechnet. Die Dauer der Versuchsperioden betrug für die Versuche mit dem Spirometer 20-30 Minuten, für die Versuche am Jaquet'schen Apparat ungefähr 1 Stunde. In den Tabellen, welche die Steigerung des Gaswechsels nach Nahrungszufuhr angeben, sind die Werte für Kohlensäure und Sauerstoff genau entsprechend der Dauer der Perioden und den Pausen berechnet (Tab. IV u. VI).

Nach Zufuhr von 50 g Kasein tritt bei beiden Versuchspersonen erst in der zweiten Stunden die Steigerung des Gaswechsels deutlich hervor. Das Maximum scheint auch in dieser Zeit erreicht zu sein. Die Steigerung überdauert bei A die dritte Stunde nicht, während bei B der Nüchternwert erst ungefälır in der vierten Stunde erreicht ist. Bei beiden Versuchspersonen laufen Kohlensäure und Sauerstoffwerte ungefähr parallel. Der respiratorisebe Quotient gibt verhältnismässig geringe Schwankungen. Aus dem letzteren lässt sich wohl kein sicherer Schluss ziehen. Die Stickstoffausscheidung ergibt:

bei $\mathrm{A}$ im Mittel nach Kasein . . 1,08 g pro Stunde

" A nüchtern. . . . . . . $0,57 ", " ~$
B im Mittel nach $50 \mathrm{~g}$ Kasein $1,06 ", "$
B nüchtern. . . . . . . $0,59 ", "$,

Die absolute Steigerung beträgt bei $\mathrm{A}$ im Mittel $5,04 \mathrm{~g} \mathrm{CO}_{2}$ und 4,87 $\mathrm{g} \mathrm{O}_{2}$, bei $\mathrm{B} 5,19 \mathrm{~g}_{\mathrm{CO}_{2}}$ und $6,03 \mathrm{~g} \mathrm{O}_{2}$. Aus diesem Vergleich ergibt sich, dass die Kaseinzufuhr von den beiden Individuen 
vollkommen gleich verwertet worden ist. Es sei hier nochmals betont, dass die beiden Personen in Länge und Gewicht nicht bedeutend differieren.

Unter gleichen Bedingungen scheint also bei gesunden Individuen die Zufuhr von $50 \mathrm{~g}$ Kasein die gleiche Gaswechselsteigerung zu bedingen.

Eine Berechnung der Eiweiss- resp. Fett- und Kohlehydratverbrennung ist hier nicht ausgeführt worden, da dieselbe, wenn die Ergebnisse von $\operatorname{Gig}_{0} \mathrm{n}^{1}$ ) richtig sind, keine zuverlässigen Resultate geben.

Die Edestinversuche führten zu folgenden Zahlen:

Tabelle VII.

Gaswechsel nach Zufuhr von $50 \mathrm{~g}$ Edestin pro ${ }^{1 / 2}$ Stunde angegeben.

\begin{tabular}{|c|c|c|c|c|c|}
\hline Datum & Zeit & $\begin{array}{l}\mathrm{CO}_{2} \text {-Aus- } \\
\text { scheidung } \\
\mathrm{g}\end{array}$ & $\begin{array}{l}\mathrm{O}_{\mathrm{g}} \text {-Ver- } \\
\text { brauch } \\
\quad \mathrm{g}\end{array}$ & $\frac{\mathrm{CO}_{2}}{{\overline{O_{2}}}_{2}}$ & $\begin{array}{c}\text { Versur'is- } \\
\text { persom }\end{array}$ \\
\hline $\begin{array}{l}\text { 8. }-9 . \text { Dez. } 1911 \\
4,97 \mathrm{~g} \mathrm{~N} \text { in } \\
4 \text { Stunden }\end{array}$ & 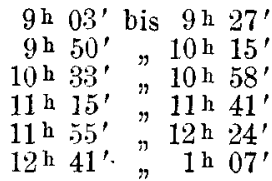 & $\begin{array}{r}10,77 \\
11,29 \\
10,94 \\
11,49 \\
9,61 \\
9,55\end{array}$ & $\begin{array}{r}9,67 \\
10,20 \\
10,00 \\
9,78 \\
8,64 \\
9,12\end{array}$ & $\begin{array}{l}0,811 \\
0,805 \\
0,795 \\
0,853 \\
0,809 \\
0,815\end{array}$ & A \\
\hline
\end{tabular}

Steigerung des Gaswechsels nach Znfuhr ron 50 g Edestin.

\begin{tabular}{|c|c|c|c|c|}
\hline Datum & Zeit & $\begin{array}{l}\mathrm{CO}_{2} \text {-Aus- } \\
\text { scheidung } \\
\mathrm{g}\end{array}$ & $\begin{array}{c}\mathrm{O}_{2-} \\
\text { Verbrauch } \\
\mathrm{g}\end{array}$ & $\begin{array}{l}\text { Versuch } \\
\text { person }\end{array}$ \\
\hline 8.-9. Dez. 1911 & 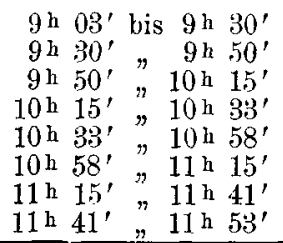 & $\begin{array}{l}9,693 \\
7,360 \\
9,400 \\
6,07 \\
9,12 \\
6,35 \\
9,93 \\
5,45\end{array}$ & $\begin{array}{l}8,69 \\
6,62 \\
8,50 \\
6,06 \\
8,34 \\
5,61 \\
8,48 \\
4,40\end{array}$ & A \\
\hline Daner . . & $\begin{array}{l}2 \text { Stunden } 50 \text { Min. } \\
\text { Nïchternwerte } \\
\text { Steigerung. . }\end{array}$ & $\begin{array}{r}68,37 \\
58,65 \\
+4,72\end{array}$ & $\begin{array}{r}56,70 \\
58,55 \\
+\mathbf{3}, 15\end{array}$ & \\
\hline
\end{tabular}

1) Gigon, Über den Einfluss der Nahrungsaufnahme auf den Gaswechsel and Energieumsatz. Pflüger's Arch. Bd.140 S. 1. 1911. 
Respirationsversuche an Menschen im nüchternen Zustand etc.

Tabelle VIII.

Gaswechsel pro Stunde nach Zufuhr ron 50 g Edestin.

\begin{tabular}{|c|c|c|c|c|c|}
\hline Datum & Zeit & $\begin{array}{c}\mathrm{CO}_{2} \text {-Aus- } \\
\text { scheidung } \\
\mathrm{g}\end{array}$ & $\begin{array}{l}\mathrm{O}_{2} \text {-Ver- } \\
\text { brauch } \\
\mathrm{g}\end{array}$ & $\frac{\mathrm{CO}_{2}}{\mathrm{O}_{2}}$ & $\begin{array}{l}\text { Versuchs- } \\
\text { person }\end{array}$ \\
\hline $\begin{array}{l}\text { 22. - 23. Sept. } \\
1911 \\
\left(\begin{array}{c}\mathrm{N}=0,96 \% \\
6,62 \mathrm{~g})\end{array}\right.\end{array}$ & 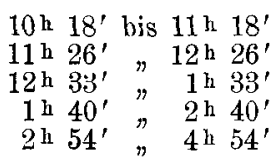 & $\begin{array}{l}23,06 \\
23,79 \\
25,85 \\
24,63 \\
23,14\end{array}$ & $\begin{array}{l}20,1 \\
21,77 \\
22,94 \\
21,91 \\
20,52\end{array}$ & $\begin{array}{l}0,831 \\
0,792 \\
0,817 \\
0.832 \\
0,835\end{array}$ & B \\
\hline
\end{tabular}

Steigerung des Gaswechsels nach Zufuhr von 50 g Edestin.

\begin{tabular}{|c|c|c|c|c|}
\hline Datum & Zeit & $\begin{array}{l}\mathrm{CO}_{2} \text {-Aus- } \\
\text { scheidung } \\
\mathrm{g}\end{array}$ & $\begin{array}{c}\mathrm{O}_{2^{-}} \\
\text {Verbrauch } \\
\quad \mathrm{g}\end{array}$ & $\begin{array}{l}\text { Versuchs- } \\
\text { person }\end{array}$ \\
\hline 22.-23. Sept. & 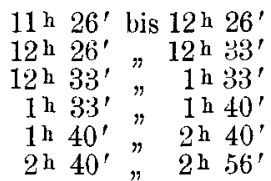 & $\begin{array}{r}23,79 \\
2,93 \\
25,85 \\
2,95 \\
24,63 \\
6,56\end{array}$ & $\begin{array}{r}21,77 \\
2,61 \\
22,94 \\
2,62 \\
21,91 \\
5,84\end{array}$ & B \\
\hline Dauer & $\begin{array}{l}33^{t / 2} \text { Stunden } \\
\text { Nüchternwert. } \\
\text { Steigerung . . }\end{array}$ & $\begin{array}{r}86,71 \\
81,75 \\
+4,96\end{array}$ & $\begin{array}{r}77,69 \\
73,69 \\
+\mathbf{4}, 00\end{array}$ & \\
\hline
\end{tabular}

Die Edestinversuche ergaben folgende Resultate: Die Steigerung des Gaswechsels ist in der ersten Stunde sehr gering (Versuchsperson B) resp. $=0$ (Versuchsperson A). Das Maximum der Kohlensäureausscheidung fällt auf die dritte Stunde. Der respiratorische Quotient gibt auch hier keine sicheren Anhaltspunkte. Der Nüchternwert ist bei A nach ca. 3 Stunden erreicht, bei B nach $3^{1 / 2}$ Stunden. Die absolute Steigerung ist bei beiden Personen etwas niedriger, wenn auch nur unbedeutend als nach Kaseinzufuhr.

Die Resultate dieser Versuche deuten mit ziemlicher Sicberheit darauf hin, dass bei verschiedenen Individuen die Zufuhr verschiedener Eiweisskörper die gleichen Veränderungen im Gaswechsel verursacht. Die Individualität, deren Bedeutung für solche Untersuchungen sehr oft hervorgehoben wurde, scheint bei gleicher zweckmässiger Versuchsanordnung keine schwerwiegenden Unterschiede hervorzurufen. Diese Schlussfolgerung ist nicht ohne Wert, da die meisten Arbeiten mit Gaswechselapparaten aus leicht verständlichen Gründen jeweils nur bei wenigen Individuen ausgeführt werden können. 
Auffallend ist nun, dass $50 \mathrm{~g}$ Kasein die gleichen Veränderungen verursachen wie $50 \mathrm{~g}$ Edestin. Beide Male ist die Dauer der Steigerung gleich, bei A 2 Stunden 50 Minuten, bei B 31/2 Stunden. Die absolute Steigerung ergibt keine wesentlichen Unterschiede. Die auffallenden Resultate bei Zufuhr verschiedener Kohlehydrate scheinen eher darauf hinzudeuten, dass auch bei verschiedenen Eiweisskörpern differierende Gaswechselwerte zu erwarten gewesen wären.

Bej den angeführten Kohlehydratversuchen wurden die letzten Abbauprodukte der Polysaccharide (Dextrose, Lävulose) untersucht. Es ist vielleicht zu erwarten, dass die Bausteine des Eiweissmoleküls, analog der Wirkung von Dextrose und Lävulose, verschiedenen Einfluss auf die Steigerung des respiratorisehen Gaswechsels ausüber können, während das Eiweissmolekül als Ganzes analoge Wirkung hätte wie die Moleküle der verschiedenen Stärkearten.

Zur Entscheidung dieser Frage haben wir Versuche mit reinen Aminosäuren begonnen.

\section{IV.}

Die Steigerung des Gaswechsels bei Eiweisszufuhr kann unmöglich auf die Verdauungsarbeit zurückgeführt werden. Die Versuche mit Strohfütterung beim Pferd von Zuntz und $\operatorname{Hagemann}^{1}$ ), sowie die Knochenfütterung bei Hunden durch Magnus-Levy ${ }^{2}$ ) scheinen allerdings darauf hinzuweisen, dass bei sehr grossen Mengen, "die über das zulässige Maass wohl herausgehen", durch mechanische Darmreizung kein vermehrter Umsatz hervorgerufen werden kann. Auch die schönen Versuche Cohnheim's ${ }^{3}$ ) mit Scheinfütterung scheinen für den Einfluss der „Verdauungsarbeit" auf den Gaswechsel $\mathrm{zu}$ sprechen; in diesen Versuchen fällt ihr Betrag jedoch sehr klein aus. Heilner ${ }^{4}$ ) kommt durch seine Untersuchungen dazu, den Einfluss der Verdauungsarbeit völlig in Abrede zu stellen. Er möchte diesen Begriff, der leicht zu Verwechslungen Anlass gibt, möglichst eliminieren. Untersuchungen verschiedener Autoren, Magnus-

1) Zuntz und Hagemann, Untersuchungen über den Stoffwechsel des Pferdes bei Rube und Arbeit. Berlin 1908.

2) Magnus-Levy, Über die Grösse des respiratorischen Gaswechsels. Pflüger's Arch. Bd. 55 S. 81. 1894.

3) Cohnheim, Der Energieaufwand bei der Verdauungsarbeit. Arch. f. Hygiene Bd. 57 S. 401. 1906.

4) Heilner, Zur Frage der Verdauungsarbeit. Zeitschr. f. Biol. Bd. 50. 1908. 
Levy ${ }^{1}$ ), Koraen ${ }^{2}$ ), Gigon ${ }^{3}$ ), ergaben, dass eine manchmal bedeutende Fettzufuhr keine Steigerung im Respirationsstoffwechsel hervorruft, obgleich dieselbe wohl ebensoviel Darmdrüsenarbeit zu ihrer Aufnahme verlangt wie $50 \mathrm{~g}$ Dextrose oder $50 \mathrm{~g}$ reiner Eiweisskörper. Im gleichen Sinne lässt sich verwerten, dass J o h an n s son bei Individuen im Nüchternzustand, d. h. bei Glykogenvorrat, eine Steigerung von $+6 \mathrm{~g} \mathrm{CO}_{2}$, beim gleichen Individuum im Hungerzustand, d. h. bei Glykogenmangel, keine Steigerung beobachtete. Endlich spricht auch dafür, dass Lävulose eine doppelt so grosse Steigerung des Gaswechsels bedingt wie die gleiche Dosis Glykose.

Wenn auch ein gewisser Einfluss der Darmtätigkeit (Drüsensekretion, Peristaltik) auf den Gaswechsel nicht geleugnet werden soll, kann derselbe zur Deutung unserer Resultate doch nicht herangezogen werden. Die notwendige Darmdrüsenfunktion, welche $50 \mathrm{~g}$ Kasein resp. Edestin zu ihrer Resorption gebrauchen, ist sehr wahrscheinlich nicht höher als die "Verdauungsarbeit", die schon im Nüchternzustand vor sich geht ${ }^{3}$ ). (Die Sekretbildung der grossen Verdauungsdrüsen erfolgt ja bekanntlich auch im Nüchternzustand.) Ihr Beitrag an den Gaswechsel ist daher schon in dem nach unserer Versuchsanordnung bestimmten Nüchternwert enthalten. Die von uns erhaltene Steigerung nach Eiweisszufuhr lässt sich nur mit den weiteren Veränderungen der Nahrungsstoffe nach ihrer Resorption aus dem Darmkanal in Zusammenhang bringen. Wir wissen, dass die Resorption sehr sehnell ${ }^{4}$ ) von statten geht, und dass nach ca. 4-6 Stunden die ganze Nahrung, wenn sie nicht zu reichlich ist und zu grosse Ansprüche an den Verdauungsapparat stellt, schon in den Dickdarm gelangt ist. Die intermediären Prozesse, welche die Nahrungsstoffe im Organismus durchmachen, beginnen wohl gleich nach der Resorption, und diese erfolgt, sobald die Speisen ins Duodenum gelangt sind. Haben die Ingesta den Dünndarm passiert, so wird bekanntlich aus dem Coecum und Colon kein wesentliches Nährmaterial mehr aufgesaugt. Die Steigerung durch Kasein kann also wohl auf intermediäre Prozesse zurückgeführt werden. Die jetzt noch

1) Magnus-Levy, l. c. S. 39.

2) Koraen, Über den Einfluss der Nahrungsaufnahme auf den Stoffwechsel. Skandin. Arch. f. Physiol. Bd. 11. 1901.

3) Gigon, l. c.

4) Magnus-Levy, v. Noorden's Handb. Bd. 1. 
am meisten vertretene Ansicht ist die, dass die Eiweisskörper gleich nach der Resorption in einem minimalen Betrage zu Körpereiweiss umgewandelt werden; der Rest würde dagegen bis zu den physiologischen Endprodukten (Harnstoff, Harnsäure) verbrannt. Die dabei freigewordene Wärme tritt teilweise an Stelle der sonst durch Körperkohlehydrat (Glykogen) resp. Körperfett produzierten Wärme; ein Teil der Wärme würde sich endlich noch als mehr oder weniger überschüssige Kalorienproduktion bemerkbar machen. [„Spezifischdynamische Wirkung" Rubner ${ }^{1}$ ).]

Fettbildung resp. Glykogenbildung würden nach dieser Anschauung nur in besonderen Fällen vorkommen, z. B. im ausgesprocbenen Hungerzustand oder unter gewissen pathologischen Bedingungen, z. B. beim Diabetes. Nach seinen neuen Untersuchungen glaubt nun Gigon ${ }^{2}$ ) eine andere Hypothese aufstellen zu können. Nach ihm sollen auch im normalen Zustand aus dem Nahrungseiweiss sowohl Glykogen wie auch Fett gebildet werden.

Die Steigerung des Gaswechsels nach Ejweisszufuhr würde auf diese beiden Prozesse zurückzuführen sein. Den Beweis dieser Annahme sucht der Autor damit zu erbringen, dass bei Variation der Grösse der Eiweissdosen im Verhältnis von $1: 2: 3: 4$ der Gaswechsel eine Zunahme im Verhältnis von 1:3:6:9 aufweist. Ist diese Anschauung richtig, so würden sowohl aus Kasein wie aus Edestin unter normalen Verhältnissen Körperglykogen und Körperfett gebildet.

Erwähnenswert, namentlich in Anbetracht der verschiedenen chemischen Zusammensetzung dieser beiden Eiweisskörper, ist es, dass anscheinend aus. diesen beiden Stoffen die gleichen Mengen Glykogen resp. Fett im Organismus gebildet werden können. Dieser Ansicht entsprechend würden Edestin und Kasein nicht unmittelbar vom Organismus verwertet. Diese Stoffe werden vielmehr zu einem grossen Teil umgewandelt, als "Reservestoffe" abgelagert, und erst als solche werden sie zur Energieproduktion verwendet.

Zugunsten dieser Anschaunng können wir die ganz auffallende Konstanz des Nüchternwertes mehrere Jahre hindurch bei verschiedenen Individuen anführen. Dafür spricht auch die exakte Proportionalität, die man für die Zunabme des Gaswechsels erhält, bei proportional steigenden Dosen von Eiweiss oder Koblehydraten.

1) Rubver, Die Gesetze des Energicverbrauchs bei der Ernährung.

2) Gigon, l. c. 


$$
\text { im Verbältnis von } \quad \text { im Verhältnis von }
$$

für Kohlehydrate. . $1: 2: 3: 4$ bedingen $1: 2: 3: 4$

"Eiweisskörper. . 1:2:3:4 " $1: 3: 6: 9$

Ein regelmässiger Nebenbefund lässt sich in allen unseren Versuchen erkennen, nämlich eine geringgradige Erniedrigung des Gaswechsels unter den Nüchternwert, kurz nachdem die durch Nahrungszufuhr bedingte Steigerung aufgehört hat. Diese Beobachtung wurde schon von Johannsson und Gig on erwähnt. Das gleiche fand Jaqu et ${ }^{1}$ ) nach der durch Muskelarbeit hervorgerufenen Steigerung des Gaswechsels. Eine Deutung dieser Anschauung lässt sich momentan nicht geben.

Unsere Resultate lassen sich noch für gewisse Fragen der vegetabilischen Diät verwerten. Es ist bekannt, dass das Eiweiss, das in der vegetarischen Kost zugeführt wird, weniger gut ausgenutzt wird als das animalische Eiweiss. Nach unserem Ergebnis dürfte nun dieser Unterschied nicht auf der Eiweisskomponente selbst beruhen, sondern vielmehr auf der Art und Weise, wie das Eiweiss, in Zellulose eingebettet, zugeführt wird. Dies geht auch aus den schönen Untersuchen von Albertoni und Rossi ${ }^{2}$ ) hervor. Diese Autoren untersuchten bei den fast vegetarisch sich ernährenden Bauern der Abruzzen die Wirkung der vegetarischen und der animalischen Kost. Sie fanden, dass bei Fleischkost auch eine vollständigere Resorption des Pflanzeneiweisses erfolgt, und dass der grosse Stickstoffverlust bei vegetarischer Ernährung auf der übermässigen Arbeit beruht, die dem Darm zugemutet wird.

Ein prinzipieller Unterschied zwischen animalischem und vegetabilischem Eiweiss scheint nicht vorzuliegen. Daraus lässt sich schliessen, dass bei entsprechender Zubereitung eine ausschliesslich vegetarische Kost leicht zuträglich ist. Die grossen Nachteile reiner Pflanzenkost, die sich nicht nur bei den freiwilligen Vegetariern der Städte, sondern auch bei jenen von der Natur zur ausschliesslichen Pflanzenkost gezwungenen Bauern gewisser Gegenden Italiens zeigen, beruhen wohl ausschliesslich auf dem enormen Schlackenreichtum dieser Kostform.

1) Jaquet, Über die Nachwirkung einer angestrengten Muskelarbeit auf den Stoffwechsel. Arch. f. exper. Pathol. u. Pharmak. Bd. 62. 1910.

2) Albertoni et Rossi, Recherches sur la valeur comparative de l'aliment végétal et de l'aliment animal et sur la bilance protéique "minimum". Arch." ital. de biol. t. 51 p. 385.1909.

Pflüger's Archiv für Physiologie. Bd. 147. 


\section{Schlusssätze.}

1. Die Resultate, die mit unserem Spirometerapparat (kurzdauernde Versuche) gewonnen werden, stimmen völlig überein mit den Ergebnissen, die mit dem Jaquet'schen und TigerstedtSondén'schen Apparat erbalten werden.

2. Der Grundumsatz blieb mehr als 4 Jahre hindurch bei der gleichen Versuchsperson konstant.

3. Bei einer Zufuhr von $50 \mathrm{~g} \mathrm{Kasein} \mathrm{(animalisches} \mathrm{Nukleo-}$ albumin) steigt der Gaswechsel um ca. $5 \mathrm{~g} \mathrm{CO}_{2}$ und ca. $5 \mathrm{~g} \mathrm{O}_{2}$. Die gleiche Zufubr von Edestin (pflanzliches Globulin) erzeugt die gleichen Gas wechselveränderungen.

4. Kasein resp. Edestin verursachen bei zwei verschiedenen Versuchsindividuen die g l e ich en Veränderungen im respiratorischen Stoffwechsel.

Zum Schluss sei es mir gestattet, Herrn Professor Eg ger meinen herzlichen Dank auszusprechen für die freundliche Überlassung des Laboratoriums und der Mittel der Poliklinik; ebenso Herrn Dr. Gigon für die Anregung zu dieser Arbeit, sowie für seine unermüdliche Unterstutzung bei der Ausführung der Versuche. 